\title{
Lipoprotein lipase gene PvuII polymorphism serum lipids and risk for coronary artery disease: Meta-analysis
}

\author{
Penbe Cagatay ${ }^{\mathrm{a}}$, Belgin Susleyici-Duman ${ }^{\mathrm{b}, *}$ and Cavlan Ciftci ${ }^{\mathrm{c}}$ \\ ${ }^{a}$ Istanbul University, Cerrahpasa Faculty of Medicine Department of Biostatistics, Istanbul, Turkey \\ ${ }^{\mathrm{b}}$ Istanbul Science University Medical Faculty, Department of Medical Biology and Genetics, Istanbul, Turkey \\ ${ }^{\mathrm{c}}$ Istanbul Science University Medical Faculty, Department of Cardiology, Istanbul, Turkey
}

\begin{abstract}
Our aim was to determine whether lipoprotein lipase gene PvuII polymorphism can be considered as an independent risk factor for coronary artery disease (CAD) by conducting a meta-analysis of all available published trials, including our own study. In 7 seperate studies, 3289 subjects were screened for this substitution; meta-analysis included only some of these individuals. Among the 7 studies, 6 were performed on white subjects, whereas 1 was on patients with Saudi Arabic descent.Subgroup analysis indicated that individuals with PvuII substitution does not have an increased risk for CAD. The LPL-PvuII genotype and allele frequency distributions did not differ significantly between CAD patients and healthy controls. There was no difference in the distribution of LPL-PvuII genotypes between the healthy subjects and the patients with CAD. However, no significant differences in lipid variables (triglyceride and HDL-cholesterol) were determined for the PvuII polymorphisms in the patients with CAD. No significant differences were found in serum triglyceride and HDL-cholesterol levels for LPL-PvuII genotypes when the control and CAD groups were pooled. In conclusion, LPL-Pvu II polymorphism cannot be used as independent genetic risk factor for CAD.
\end{abstract}

Keywords: Lipoprotein lipase, coronary artery disease, genetic polymorphism, meta-analysis

\section{Introduction}

In the present study we have concentrated on the PvuII gene polymorphism of the lipoprotein lipase (LPL) which play an important role in the lipid metabolism [1]. Because polymorphism studies in large groups of human subjects are laboraous, each study permits only a limited number of comparisons and limited power to obtain conclusive results on genotype and lipid interactions. An alternative to repeating a study with a large sample size is to combine data

\footnotetext{
*Corresponding author: Assoc. Prof. Belgin Susleyici-Duman, Istanbul Science University Medical Faculty, Department of Medical Biology and Genetics, Büyükdere Street No.120, Esentepe-Istanbul, Turkey. Tel.: +90 21221364 83; Fax: +90 21227234 61; E-mail: belgin.susleyici@bilim.edu.tr.
}

from other studies. The technique of testing the statistical significances of combined data is commonly called meta-analysis. We carried out such an analysis of several LPL gene Pvu II polymorphism studies who worked on coronary artery disease patients by pooling the genotype effects and significances into overall estimates and then testing for combined significance.

The aim of the present study was to look for the possible associations between the LPL PvuII gene polymorphism and the lipid levels in the patients with CAD. Since a meta-analysis has been published examining the effects of 4 amino acid-changing substitutions (Gly188Glu, Asp9Asn, Asn291Ser, Ser447Ter) of the LPL on serum lipids and lipoproteins and risk of ischemic heart disease [2] we considered the PvuII polymorphism in exon 6 that does not change amino acid in lipoprotein lipase enzyme in this study. In meta 
analysis we assessed the effects of this substitution on serum lipids, and risk of CAD.

\section{Materials and methods}

\subsection{Study subjects}

The selection criteria for studies to be considered for this meta-analysis were as follows: (1) the PvuII polymorphism in LPL were studied; (2) of the 3289 participants 2347 were white whereas 942 were Saudi Arabic; (3) case-control and case-referant studies were included that identified the PvuII genotypes; (4) data were reported on at least 1 of the following variables: triglycerides, total-cholesterol, HDLcholesterol, LDL-cholesterol; (5) only data published as part of full articles in peer reviewed journals were considered; (6) criteria for CAD was narrowing of artery $50 \%$ or more with angiography; (7) four studies identified controls by the lack of CAD or other atherosclerotic cardiovascular disorders by diagnosis, one study $<10 \%$ stenosis present with angiography, and one study with completely normal angiography; (8) subjects were of unrestricted age and gender.

\subsection{Search strategy}

Literature searches through January 1970 and March 2006 included Medline search, Embase search and the reference lists of articles already on file. A total of 12 studies including 3289 subjects were included [39], whereas 2 studies [10,11] were excluded (tables available from authors). Data were collected as they appeared in the original studies.

\section{Meta-analysis}

Meta-analysis were performed on STATA 5.0 and EXCEL 5. All published reports which investigated the frequency of LPL-PvuII DNA polymorphisms and their effects on serum lipids among CAD and healthy adults between 1970 and 2005 were identified by literature searches. Including our present study [9], 7 eligible reports were found [3-9]. Reports were performed on Saudi Arab population [8], European [4], 2 Turkish population [7,9], mainly Portuguese, Italian, Spanish and German [6], French [3], northern European (Anglo-Scandinavian) (genetically representative of North American Caucasian) [5]. Two studies were not included in the analysis, since, one was performed on monozygotic twins [10], whereas other study reported as geometric mean values [11]. Data on the LPL PvuII polymorphisms were available in most studies. The genotype frequencies of CAD and controls were analyzed in 4 reports [5,7-9]. The genotype frequencies between patients and control subjects were counted and compared by $\chi^{2}$ analysis in each study, then pooled and evaluated together by using the same method. Two reports [4,9] were combined to find the differences of triglyceride and total-cholesterol levels among the PvuII genotypes in patients with CAD. The TG and T-Chol levels in report 12 was converted into SI units before the analysis. Conversion factors for SI units were; division by 39 of $\mathrm{mg} / \mathrm{dl}$ to convert to $\mathrm{mmol} / \mathrm{L}$ for T-Chol and HDL-cholesterol, and division by 89 of $\mathrm{mg} / \mathrm{dl}$ to convert to $\mathrm{mmol} / \mathrm{L}$ for triglycerides. The standard error of mean (SEM) values vere converted into standard deviation (SD). In the studies where PvuII allele frequencies were not given, the genotype distributions were converted into allelic distributions. Individual studies were combined by using weighted values method in order to evaluate whether triglyceride and HDL-cholesterol levels varied as a function of PvuII genotypes. In the original report of Rios et al. the triglyceride and HDL-cholesterol levels were given seperately for men and women, for this reason the results were combined and calculated again to find the differences of triglyceride and total-cholesterol levels among the PvuII genotypes in patients with CAD and controls. The equations used for the calculation of weighted values are as follows. The triglyceride and HDL-cholesterol data obtained by this method were compared among PvuII genotypes by using one-way ANOVA.

$$
\begin{aligned}
\bar{X} & =\frac{\sum_{i=1}^{k}\left(\bar{x}_{i} * n_{i}\right)}{N} \\
S D & =\sqrt{\frac{\sum_{i=1}^{k}\left(\left(n_{i}-1\right) * S D_{i}^{2}+\left(n_{i} * \bar{x}_{i}^{2}\right)\right)-N \bar{X}^{2}}{N-1}}
\end{aligned}
$$

In the second equation; $N-1$ is used when $N$ is small or equal to 30, whereas $N$ is used when $N$ is larger than 30 .

The reports which evaluated the same parameters were pooled. For this purpose reports 4,6,9 were combined and analyzed for TG, T-cholesterol and HDLcholesterol. In order to compare the PvuII allele fre- 
Table 1

Baseline characteristics of the studies included in the meta-analysis

\begin{tabular}{llcrl}
\hline Reference & & Mean age (y) & $\mathrm{n}$ & Population \\
\hline Abu-Amero K. & CAD (men + women) & 44 & 431 & S. Arabian \\
& Control (men + women) & 37 & 511 & \\
Isbir T. & CAD (men + women) & 52.61 & 173 & Turkish \\
& Control (men + women) & 52.57 & 111 & \\
Bailly D. & CAD (men + women) & 33 to 55 & 74 & French \\
& Control (men + women) & 20 to 65 & 289 & \\
Anderson JL. & CAD (men + women) & 65 & 483 & Norh American Caucasian \\
& Control (men + women) & 61 & 168 & (North European) \\
Duman BS. & CAD (men + women) & 58.43 & 80 & Turkish \\
& Control (men + women) & 58.98 & 45 & \\
Wang XL. & CAD (men) & 55.40 & 352 & European \\
& CAD (women) & 56 & 127 & \\
Domingos LS. & CAD (men + women) & 59.10 & 309 & Mainly Portuquese, Italian, \\
& Control (men + women) & 55.30 & 197 & Spanish, German \\
\hline
\end{tabular}

CAD: Coronary artery disease.

Table 2

Lipoprotein lipase PvuII allele distributions in coronary artery disease patients and controls

\begin{tabular}{lcccccc}
\hline Reference & \multicolumn{2}{c}{$P+$} & & \multicolumn{2}{c}{$P-$} & \\
\cline { 2 - 3 } & CAD (n) & Control (n) & & CAD (n) & Control (n) nn & p \\
\hline Abu-Amero K. & 501 & 612 & & 361 & 410 & $>0.05$ \\
Isbir T. & 123 & 80 & & 77 & 64 & $>0.05$ \\
Bailly R. & 35 & 175 & & 39 & 163 & $>0.05$ \\
Anderson JL. & 520 & 196 & & 446 & 140 & $>0.05$ \\
Duman BS. & 89 & 44 & & 67 & 54 & $=0.05$ \\
Pooled Results & 1268 & 1107 & & 990 & 831 & $>0.05$ \\
\hline
\end{tabular}

CAD: Coronary artery disease.

Table 3

Lipoprotein lipase PvuII genotype distributions in coronary artery disease patients and controls

\begin{tabular}{|c|c|c|c|c|c|c|c|}
\hline \multirow[t]{3}{*}{ Reference } & \multicolumn{6}{|c|}{ LPL-PvuII genotypes } & \multirow{3}{*}{$\mathrm{p}$} \\
\hline & \multicolumn{2}{|c|}{$P+/+$} & \multicolumn{2}{|c|}{$P+/-$} & \multicolumn{2}{|c|}{$P-/-$} & \\
\hline & CAD & Control & CAD & Control & CAD & Control & \\
\hline Abu-Amero $\mathrm{K}$. & 138 & 182 & 225 & 248 & 68 & 81 & $>0.05$ \\
\hline Isbir $\mathrm{T}$. & 37 & 20 & 49 & 40 & 14 & 12 & $>0.05$ \\
\hline Anderson JL. & 142 & 60 & 236 & 76 & 105 & 32 & $>0.05$ \\
\hline Duman BS. & 25 & 14 & 39 & 16 & 14 & 19 & $<0.05$ \\
\hline Pooled Results & 342 & 276 & 549 & 380 & 201 & 144 & $>0.05$ \\
\hline
\end{tabular}

quencies of the published reports, the genotype frequencies were converted into allele frequencies in studies 3, 5, 8 and 9 .

\section{Results}

Seven case-control or case-referent studies including 3289 subjects were selected for this meta-analysis. The PvuII substitution influenced the serum totalcholesterol and LDL-cholesterol levels by 0.05 and 0.02 respectively only in one study [9]. The studies included in the meta-analysis are listed in Table 1. All the studies had been conducted on patients with CAD and healthy controls. LPL-PvuII allele distributions in CAD patients and controls are given in Table 2. The distribution of the PvuII alleles in one study [9] differed from that of other studies, because these studies have not found significant differences in allele frequencies when patient and control groups were compared. The LPL-PvuII genotype distributions in patients and controls are presented in Table 3. The studies reporting the genotype frequencies have not found significant differences between the study groups $[5,7,8]$, except one study [9], in which significant difference had been reported. When all the studies examining LPL PvuII genotypes were pooled, the genotype frequencies were 
Table 4

Triglyceride levels of lipoprotein lipase PvuII genotypes in coronary artery disease patients

\begin{tabular}{lcccr}
\hline \multirow{2}{*}{ Reference } & \multicolumn{3}{c}{ LPL-PvuII genotypes } & \multirow{2}{*}{$\mathrm{N}$} \\
\cline { 2 - 4 } & $P+/+(n)$ & $P+/-(n)$ & $P-/-(n)$ & \\
\hline Duman BS. & $1.77 \pm 0.95(25)$ & $1.56 \pm 0.68(39)$ & $1.63 \pm 0.74(14)$ & 78 \\
Wang XL. & $2.27 \pm 1.21(102)$ & $2.21 \pm 1.07(179)$ & $2.03 \pm 0.91(69)$ & 350 \\
Weighted values & $2.17 \pm 1.38(127)$ & $2.09 \pm 1.09(218)$ & $1.96 \pm 0.79(83)$ & 428 \\
\hline
\end{tabular}

Table 5

HDL-cholesterol levels of lipoprotein lipase PvuII genotypes in coronary artery disease patients

\begin{tabular}{lcccr}
\hline Reference & \multicolumn{3}{c}{ LPL-PvuII genotypes } & \multirow{2}{*}{ N } \\
\cline { 2 - 4 } & \multicolumn{1}{c}{$P+/+$} & $P+/-$ & $P-/-$ & \\
\hline Duman BS. & $1.27 \pm 0.85(25)$ & $1.01 \pm 0.24(39)$ & $1.19 \pm 0.71(14)$ & 78 \\
Wang XL. & $1.04 \pm 0.30(102)$ & $1.04 \pm 0.26(179)$ & $1.04 \pm 0.25(69)$ & 350 \\
Weighted values & $1.08 \pm 0.22(127)$ & $1.03 \pm 0.07(218)$ & $1.06 \pm 0.14(83)$ & 428 \\
\hline
\end{tabular}

Table 6

Triglyceride levels of lipoprotein lipase PvuII genotypes in coronary artery disease patients and controls

\begin{tabular}{lcccr}
\hline Reference & \multicolumn{3}{c}{ LPL-PvuII genotypes } & \multirow{2}{*}{ N } \\
\cline { 2 - 4 } & $P+/+(n)$ & $P+/-(n)$ & $P-/-(n)$ & \\
\hline Duman BS. & $1.77 \pm 0.95(25)$ & $1.56 \pm 0.68(39)$ & $1.63 \pm 0.74(14)$ & 78 \\
Wang XL. & $2.27 \pm 1.21(102)$ & $2.21 \pm 1.07(179)$ & $2.03 \pm 0.91(69)$ & 350 \\
Rios D. & $1.75 \pm 1.06(121)$ & $1.69 \pm 0.77(235)$ & $1.72 \pm 0.81(127)$ & 483 \\
Weighted values & $1.96 \pm 1.31(248)$ & $1.88 \pm 0.88(453)$ & $1.81 \pm 0.73(210)$ & 911 \\
\hline
\end{tabular}

Table 7

HDL-cholesterol levels of lipoprotein lipase PvuII genotypes in coronary artery disease patients and controls

\begin{tabular}{lcccr}
\hline Reference & \multicolumn{3}{c}{ LPL-PvuII genotypes } & N \\
\cline { 2 - 4 } & $P+/+(n)$ & $P+/-(n)$ & $P-/-(n)$ & \\
\hline Duman BS. & $1.27 \pm 0.85(25)$ & $1.01 \pm 0.24(39)$ & $1.19 \pm 0.71(14)$ & 78 \\
Wang XL. & $1.04 \pm 0.30(102)$ & $1.04 \pm 0.26(179)$ & $1.04 \pm 0.25(69)$ & 350 \\
Rios D. & $1.10 \pm 0.06(121)$ & $1.12 \pm 0.08(235)$ & $1.09 \pm 0.08(127)$ & 483 \\
Weighted values & $1.09 \pm 0.11(248)$ & $1.07 \pm 0.05(453)$ & $1.08 \pm 0.05(210)$ & 911 \\
\hline
\end{tabular}

not found to differ between coronary artery disease patients and control subjects $\left(\chi^{2}=2.19, p>0.05\right)$.

In order to find out whether PvuII genotypes had significant effect on the serum triglyceride and HDLcholesterol levels, we combined the available literature into two groups. One group of literatures only had results for the CAD patients $[4,9]$, whereas other group consisted of the results pooled for CAD patients and controls $[4,6,9]$. Literature 6 consisted of the results given seperately for both sexes, so the results for male and females were combined prior to analysis. In the CAD group, neither triglyceride $(\mathrm{F}=0.85, p>0.05)$ (Table 4) nor HDL-cholesterol $(\mathrm{F}=5.07, p>0.05)$ (Table 5) levels were not found to differ significantly between the PvuII genotypes by analysis of variance. Additionally in the pooled (CAD + control) group, neither triglyceride $(\mathrm{F}=1.32, p>0.05)$ (Table 6$)$ nor HDL-cholesterol $(\mathrm{F}=6.40, p>0.05)$ (Table 7) levels were not found to differ significantly between the PvuII genotypes by analysis of variance. As a result, the PvuII genotypes were not found to effect serum triglyceride and HDL-cholesterol levels significantly.

\section{Discussion}

The physiologic role of the LPL-PvuII polymorphism in exon 6 is still unclear. The polymorphism does not alter serum lipid concentrations, since it does not alter the amino acid sequence. Exclusion criteria of the available literature was that, the study of Gerdes et al. [10] have included the results of triglycerides as geometric mean values and adjusted HDL-cholesterol levels. Thorn et al. [11] have performed their study in monozygotic twins. Fixed and random effects models can give very different answers, and examples may be created where either model gives counterintuitive results. Usually, though, the answers provided by these 
different modeling assumptions are similar. Differences only arise when studies are not homogenous. Since the studies used in our meta-analyses were found to be homogenous, we did not expect differences between the fixed and random effects models. The present meta-analysis suggest that risk of CAD is not influenced by PvuII polymorphism. In our previously published study [9], the genotype and allele distributions of the subjects differed from that of other cohorts $[3,5$, $7,8]$. When all the studies examining LPL-PvuII genotypes were pooled, the genotype frequencies were not found to differ between CAD patients and control subjects. In the CAD group, neither triglyceride nor HDLcholesterol levels were not found to differ significantly between the PvuII genotypes by analysis of variance. Additionally in the pooled (CAD + control) group, neither triglyceride nor HDL-cholesterol levels were not found to differ significantly between the PvuII genotypes. As a result, the Pvu II genotypes were not found to effect serum TG and HDL-cholesterol levels.

Although all the studies included for this metaanalysis were of high quality and were published in peer-reviewed journals, several problems related to the individual studies may have influenced the summarized results. (1) interaction with other potentially important variables such as age, diabetes mellitus, alcohol consumption, smoking habits, body mass index, digestion of saturated versus unsaturated fat, exercise, certain medication, and menopausal status and use of hormonal replacement therapy for women may be important for the results regarding lipids. Some of the studies included in the present meta-analysis presented plasma lipid values adjusted for some or all of these variables; however, although the consequence of such adjustment may have been to remove the effect of the substitution observed for unadjusted values, aggregated data neverthless suggested that there was not a detectable effect of this polymorphism. (2) criteria for control subject identification was not similar in all studies, in which, some studies did not verify the lack of CAD by angiography [3,6-8], but instead used only diagnostic criteria. Involving control subjects without performing angiography does not completely rule out the possibility of any cardiovascular event.

\subsection{Biological mechanism}

Lipoprotein lipase is known to be organized in an $\mathrm{N}$-domain (residues 1 to 312 ), which is impotant for the catalytic function of the enzyme, and a C-domain (residues 313 to 448), which is important for the lipoprotein lipase-mediated uptake of lipoproteins by receptors on the cell surface [12]. Possibly LPL substitutions leading to decreased enzyme activity and consequently elevated triglyceride levels and reduced HDL-cholesterol levels should increase an individual's risk of coronary artery disease. Both increased plasma triglycerides and reduced HDL-cholesterol levels are known cardiovascular risk factors $[13,14]$. The exact mechanism by which a mutation in LPL should result in remnant accumulation, is not clear. Reduced HDL-cholesterol may result in reduced reverse cholesterol transport, indirectly promoting atherosclerosis [14]. Mutations in LPL resulting in increased triglyceride levels could promote atherosclerosis by by their association with small, dense LDL particles, by an influence on hemostasis or by promoting postprandial triglyceride-rich lipoproteins [13]. Besides linkage disequilibrium with other causative mutations nearby cannot be excluded.

More population based research to support the role of the PvuII substitution is required. Additionally, the potential differences related to sex and different ethnic groups, and interactions with other common polymorphisms, need to be explored.

\section{References}

[1] I.J. Goldberg, Lipoprotein lipase and lipolysis: central roles in lipoprotein metabolism and atherogenesis, J Lipid Res 37 (1996), 693-707.

[2] H.H. Wittrup, A. Tybjaerg-Hansen and B.G. Nordestgaard, Lipoprotein lipase mutations, plasma lipids and lipoproteins, and risk of ischemic heart disease. A meta-analysis, Circulation 99 (1999), 2901-2907.

[3] A. Regis-Bailly, S. Visvikis, J. Steinmetz, L. Feldmann, S. Briancon, N. Danchin, F. Zannad and G. Siest, Frequencies of five genetic polymorphisms in coronarographed patients and effects on lipid levels in a supposedly healthy population, Clin Genet 50 (1996), 339-347.

[4] X.L. Wang, R.M. McCredie and D.E. Wilcken, Common DNA polymorphisms at the lipoprotein lipase gene. Association with severity of coronary artery disease and diabetes, Circulation 93 (1996), 1339-1345.

[5] J.L. Anderson, G.J. King, T.L. Bair, S.P. Elmer, J.B. Muhlestein, J. Habashi, L. Mixson and J.F. Carlquist, Association of lipoprotein lipase gene polymorphisms with coronary artery disease, J Am Coll Cardiol 33 (1999), 1013-1020.

[6] D.L. Rios, A.F. Vargas, G.M. Ewald, M.R. Torres, A.J. Zago, S.M. Callegari-Jacques and M.H. Hutz, Common variants in the lipoprotein lipase gene in Brazil: association with lipids and angiographically assessed coronary atherosclerosis, Clin Chem Lab Med 41 (2003), 1351-1356.

[7] T. Isbir, H. Yilmaz, B. Agachan and Z.E. Karaali, Cholesterol ester transfer protein, apolipoprotein $\mathrm{E}$ and lipoprotein lipase genotypes in patients with coronary artery disease in the Turkish population, Clin Genet 64 (2003), 228-234. 
[8] K.K. Abu-Amero, C.A. Wyngaard, O.M. Al-Boudari, M. Kambouris and N. Dzimiri, Lack of association of lipoprotein lipase gene polymorphisms with coronary artery disease in the Saudi Arab population, Arch Pathol Lab Med 127 (2003), 597-600.

[9] B.S. Duman, C. Turkoglu, B. Akpinar, M. Guden, A. Vertii, E. Dak, P. Cagatay, D. Gunay and A.S. Buyukdevrim, Lipoprotein lipase gene polymorphism and lipid profile in coronary artery disease, Arch Pathol Lab Med 128 (2004), 869-874.

[10] C. Gerdes, L.U. Gerdes, P.S. Hansen and O. Faergeman, Polymorphisms in the lipoprotein lipase gene and their associations with plasma lipid concentrations in 40-year-old Danish men, Circulation 92 (1995), 1765-1769.

[11] J.A. Thorn, E.W. Needham, R.K. Mattu, J. Stocks and D.J.
Galton, The Ser447-Ter mutation of the lipoprotein lipase gene relates to variability of serum lipid and lipoprotein levels in monozygotic twins, J Lipid Res 39 (1998), 437-441.

[12] V. Murthy, P. Julien and C. Gagne, Molecular pathobiology of the human lipoprotein lipase gene, Pharmacol Ther 70 (1996), $101-135$.

[13] J.E. Hokanson and M.A. Austin, Plasma triglyceride level is a risk factor for cardiovascular disease independent of high-density lipoprotein cholesterol level: a meta-analysis of population-based prospective studies, J Cardiovasc Risk 3 (1996), 213-219.

[14] P.J. Barter and K.A. Rye, High density lipoproteins and coronary heart disease, Atherosclerosis 121 (1996), 1-12. 


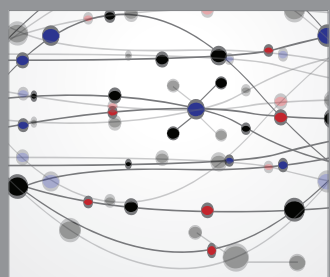

The Scientific World Journal
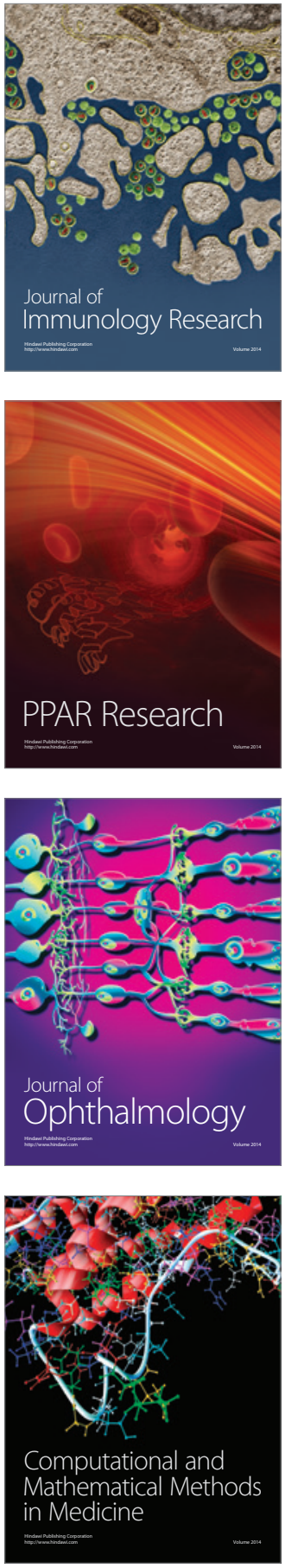

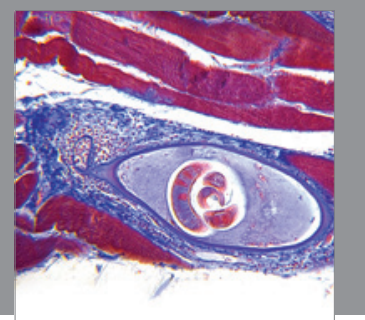

Gastroenterology

Research and Practice
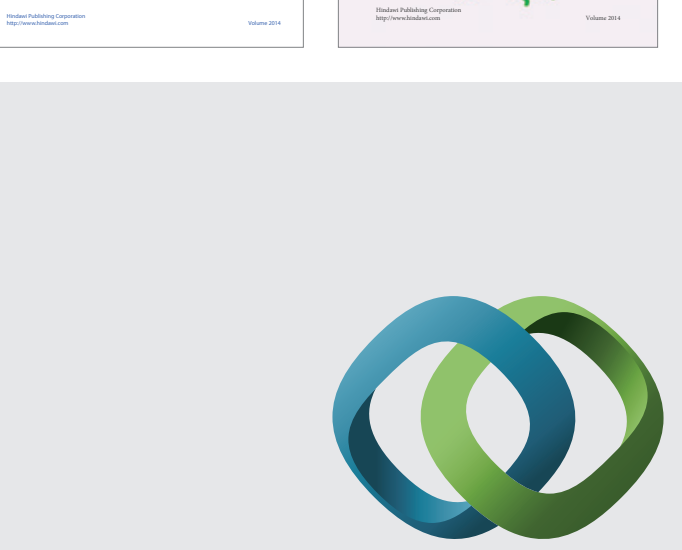

\section{Hindawi}

Submit your manuscripts at

http://www.hindawi.com
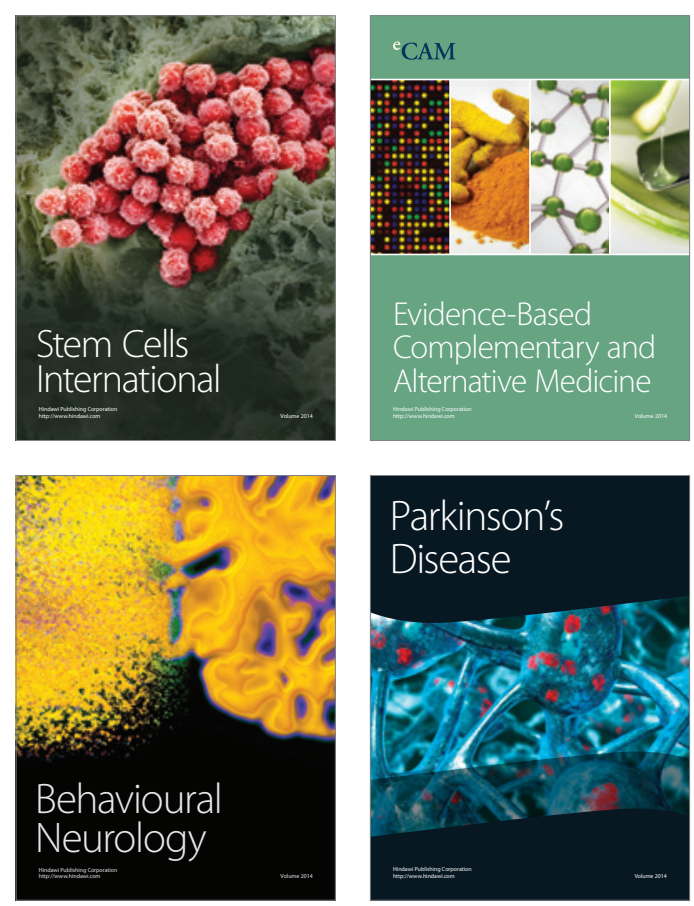

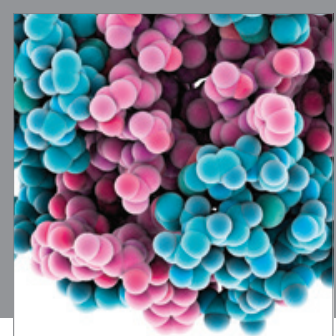

Journal of
Diabetes Research

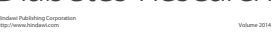

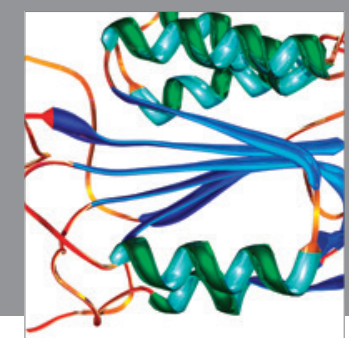

Disease Markers
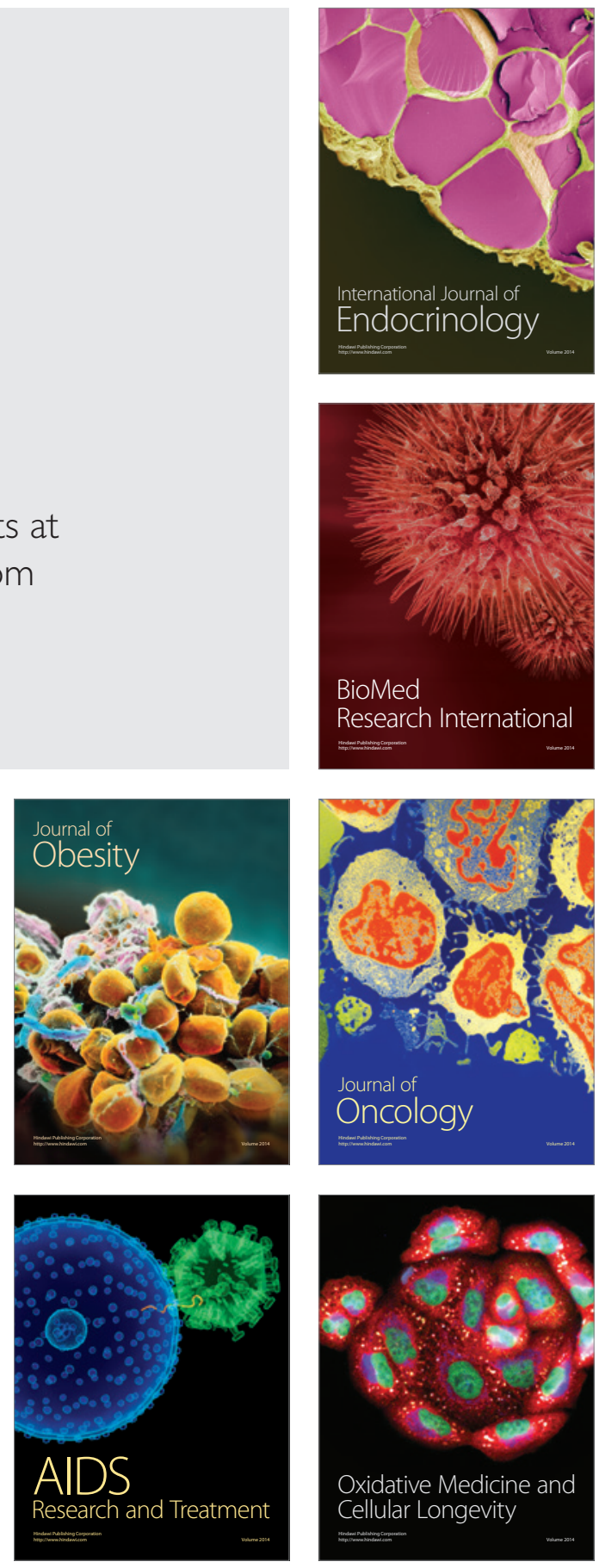